\title{
Magnetic Fe doped ZnO nanofibers obtained by electrospinning
}

\author{
Anna Baranowska-Korczyc • Anna Reszka $\cdot$ Kamil Sobczak • Bożena Sikora • \\ Piotr Dziawa · Marta Aleszkiewicz • Lukasz Kłopotowski • Wojciech Paszkowicz • \\ Piotr Dłużewski • Bogdan J. Kowalski - Tomasz A. Kowalewski • Maciej Sawicki • \\ Danek Elbaum $\cdot$ Krzysztof Fronc
}

Received: 8 August 2011/Accepted: 5 December 2011/Published online: 17 December 2011

(C) The Author(s) 2011. This article is published with open access at Springerlink.com

\begin{abstract}
We demonstrate structural and room temperature magnetic properties of Fe doped $\mathrm{ZnO}$ nanofibers (NFs) obtained by electrospinning followed by calcination. The observed NFs, formed from crystalographically oriented, approximately $4.5 \mathrm{~nm}$ particles conglomerates, were approximately $200 \mathrm{~nm}$ in diameter. The reported synthesis of room temperature ferromagnetic Fe doped $\mathrm{ZnO}$ NFs is both facile and economical, and is therefore suggested as a generic method of fabricating biocompatible magnetic materials. The major substrates selected for the NFs synthesis $(\mathrm{Zn}, \mathrm{Fe})$ comprised of relatively low toxicity materials. Incorporating $10 \% \mathrm{Fe}$ into $\mathrm{ZnO}$ does not modify the wurtzite crystal structure of the host material. No evidence of impurity phase was detected by either X-ray or electron diffraction. Magnetometry studies and Magnetic Force Microscopy imaging reveal a local ferromagnetic order that persists up to room temperature. We suggest that the observed phenomenon is either due to a mechanism mediated by presence of oxygen vacancies and/or is related to iron-rich precipitates.
\end{abstract}

Keywords Electrospinning $\cdot \mathrm{ZnO}$ nanofibers $\cdot \mathrm{ZnFeO}$. Room temperature ferromagnetism $\cdot$ Magnetic oxides

\footnotetext{
A. Baranowska-Korczyc $(\bowtie) \cdot$ A. Reszka $\cdot$ K. Sobczak B. Sikora · P. Dziawa · M. Aleszkiewicz - Ł. Kłopotowski · W. Paszkowicz · P. Dłużewski · B. J. Kowalski · M. Sawicki · D. Elbaum · K. Fronc Institute of Physics, Polish Academy of Sciences, al. Lotników 32/46, 02668 Warsaw, Poland

e-mail: akorczyc@ifpan.edu.pl

T. A. Kowalewski

Institute of Fundamental Technology Research, Polish Academy of Sciences, ul. Pawińskiego 5B, 02106 Warsaw, Poland
}

\section{Introduction}

The physical origin and the occurrence of $\mathrm{ZnO}$ doped with transition metal cations ferromagnetism are subject to active research. In spite of numerous studies the ferromagnetic properties of transition metal cations doped wurtzite $\mathrm{ZnO}$ host is still a controversial issue [1-6]. A high temperature ferromagnetism was reported numerously for $\mathrm{Co}$ doped $\mathrm{ZnO}$ in thin films produced by all accessible methods including pulsed layer deposition [7], the magnetron co-sputtering [8], the solgel method [9], and epitaxial thin films [10]. Recently, Fe doped $\mathrm{ZnO}$ nanostructures, synthesized by the sol-gel method, were observed to be ferromagnetic [11]. The observed room temperature ferromagnetism was reported to be an intrinsic $\mathrm{Fe}$ doped $\mathrm{ZnO}$ property. Interestingly, neither $\mathrm{Fe}$, nor $\mathrm{FeO}$ and $\mathrm{Fe}_{2} \mathrm{O}_{3}$ phase in the $\mathrm{Fe}$ doped $\mathrm{ZnO}$ nanoparticles were observed based on X-ray absorption near edge structure measurements [11]. On the other hand, there are report claiming no essential role of the transition metals in the $\mathrm{ZnO}$ magnetism in low dimension $\mathrm{Fe}$ and $\mathrm{Mn}$ doped $\mathrm{ZnO}$ films [12]. In particular, based on the magnetic properties of $\mathrm{Mn}$ doped $\mathrm{ZnO}$ layers, after subtraction of the substrate effects, no evidence of a sample ferromagnetism was observed [13]. Both reports point that a significant contribution to the room temperature ferromagnetism comes from the surface and/or the interface defects located between the sample and substrate. Interestingly, ferromagnetism has been also reported in thin films and nanoparticles of a large family of undoped wide gap semiconductors $\left(\mathrm{ZnO}, \mathrm{TiO}_{2}, \mathrm{HfO}_{2}, \mathrm{SnO}_{2}\right.$, etc.) [3]. The ferromagnetism observed in the semiconductor nanostructures was attributed to oxygen defects which are of special significance in low dimension samples [14]. Additionally, based on a study of laser-deposited thin films of $\mathrm{Zn}_{1-\mathrm{x}} \mathrm{Co}_{\mathrm{x}} \mathrm{O}$, the antiferromagnetic super exchange interaction was proposed to be the dominant mechanism for the exchange coupling between $\mathrm{Co}$ 
and $\mathrm{Zn}_{1-\mathrm{x}} \mathrm{Co}_{\mathrm{x}} \mathrm{O}$ [15]. Similar explanation was provided for polycrystalline powders synthesized by two different methods: solid-state and liquid-phase reactions [16]. The above observations have their foundation in calculations of the local spin density functional theory for $\mathrm{Zn}_{1-x} \mathrm{Co}_{\mathrm{x}}$ [6]. The authors observed that the dominant magnetic interaction in wellcharacterized stochiometric phase ( $\mathrm{x}$ up to 0.15 ) is dominated by the near neighbour antiferromagnetic coupling and these calculations suggested that ferromagnetism will only occur in the presence of additional hole doping. Based on similar calculations a short range nature of ferromagnetic and antiferromagnetic interactions was observed and a spin-glass like state was noted based on energy considerations for Co doped $\mathrm{ZnO}$ [17]. Recently, defect induced $\mathrm{Fe}$ doped $\mathrm{ZnO}$ reversible ferromagnetism was reported and attributed to hydrogen mediated oxygen vacancies [18]. Similarly, ferromagnetic Co doped $\mathrm{ZnO}$ was reported and reversible manipulation of shortrange spin ordering by injection and ejection of hydrogen was demonstrated [19].

1D semiconductor nanostructures such as wires, tubes, belts and fibers have attracted a great deal of attention in recent years. Among many techniques, which allow to obtain 1D semiconductors, electrospinning is one of the most simple, versatile, low-cost, and effective. The method has an attractive potential for producing NFs from polymers, composites or ceramics with diameters ranging from tens of nanometers to microns [20-24]. Moreover, NFs length is limited only by the stability of process parameters, time, and amount of applied material [23, 24]. In particular, electrospinning, followed by calcination, is a versatile way to synthesize $\mathrm{ZnO}$ nanofibers [25-27]. The nanofibers consist of conglomerates of nanostructured polycrystalline domains, which are responsible for their very large surface to volume ratio. $\mathrm{ZnO}$ doped with transition metal ions such as $\mathrm{Co}, \mathrm{Mn}, \mathrm{Ni}$ or $\mathrm{Fe}$ is a promising candidate for a room temperature ferromagnetic semiconductor [28]. Additionally, combining the ability to form NFs with transition metal doping makes the electrospinning a very promising method for providing magnetic nanofibers with potential applications in magnetic sensors, flexible magnets and biosensors.

In this study, we demonstrate the feasibility of obtaining $\mathrm{Fe}$ doped $\mathrm{ZnO}$ nanofibers exhibiting ferromagnetism above room temperature. We show that the ferromagnetic signal derives from a collective behavior typical for a blocked super paramagnet and estimate the size of nano crystallites which form the blocked state. We point out that incorporating $10 \% \mathrm{Fe}$ into $\mathrm{ZnO}$ in electrospun materials does not lead to sizeable precipitates of other impurity phases and discuss possible origins of the ferromagnetic coupling. The synthesized material is non toxic thus could have potential biomedical applications [29-31].

\section{Experimental}

\subsection{Electrospinning and calcinations}

We prepared undoped and iron doped $\mathrm{ZnO}$ nanofibers by a procedure, which consisted of four steps. The first step was to obtain $10 \%$ (wt) solution of poly(vinyl alcohol) (PVA, $\left.\mathrm{M}_{\mathrm{W}}=72,000\right)\left(\mathrm{C}_{2} \mathrm{H}_{4}\right)_{\mathrm{n}}$ (POCH, Poland) in deionized water $(0.08 \mu \mathrm{S})$. Aqueous solution of PVA was prepared by keeping the mixture at $60{ }^{\circ} \mathrm{C}$ for 7 days. The second step was a sol-gel process, $0.25 \mathrm{~g}$ of zinc acetate dihydrate $\left(\mathrm{Zn}\left(\mathrm{CH}_{3} \mathrm{COO}\right)_{2} \cdot 2 \mathrm{H}_{2} \mathrm{O}\right)$ (CHEMPUR, Poland) was dissolved in $1.25 \mathrm{~g}$ solution of PVA as a precursor to obtain $\mathrm{ZnO}$ nanofibers. Fe doping was realized by adding $0.022 \mathrm{~g}$ iron acetate $\left(\mathrm{CH}_{3} \mathrm{CO}_{2}\right)_{2} \mathrm{Fe}$ (Sigma Aldrich) to the mixture. The atomic ratio of $\mathrm{Fe}$ to $\mathrm{Zn}$ ions was about 0.1. The following step was the electrospinning process. The flow rate was stabilized at $0.2 \mathrm{ml} / \mathrm{h}$ by a syringe pump (Ascor S.A., AP12). The electric potential applied between the tip of the needle and the grounded collector placed $15 \mathrm{~cm}$ below was equal to $30 \mathrm{kV}$. In order to produce uniaxially oriented fibers for magnetometric studies, we employed a collector consisting of two separated bars of a conductive material [32]. The fibers were formed at $22{ }^{\circ} \mathrm{C}$ and humidity in the range from 30 to $40 \%$. The last step to form $\mathrm{ZnO}$ was the NFs calcination in air, at $500{ }^{\circ} \mathrm{C}$ for $4 \mathrm{~h}$.

\subsection{Characterization}

Structural characterization of NFs was performed by X-ray diffraction (XRD) and transmission electron microscopy (TEM). A high-resolution powder diffractometer (Philips X'Pert MPD Pro Alpha1) employing $\mathrm{CuK} \alpha_{1}$ radiation was used. The Bragg-Brentano geometry of the apparatus was modified by monochromatizing the incident beam using a Johansson Ge (111) monochromator. For detection, a linear semiconductor strip detector was used. The data were collected for $12 \mathrm{~h}$. TEM and electron diffraction images were obtained on a JEM2000EX electron transmission microscope at an accelerating voltage of $200 \mathrm{kV}$.

Scanning electron microscopy (SEM) provided information on NFs morphology. SEM images were obtained on Hitachi SU-70 microscope at an accelerating voltage of $5 \mathrm{kV}$. Chemical composition was investigated by energy dispersive X-ray spectroscopy (EDX) with incident beam current $6.5 \mathrm{nA}$ and accelerating voltage $15 \mathrm{kV}$ (Thermo Scientific Ultra Dry). Light emission properties were probed by cathodoluminescence (CL) (Gatan MonoCl-3) with beam accelerating voltage of $15 \mathrm{keV}$ and incident current of $6.5 \mathrm{nA}$. To gain general information about the magnetic properties, the $\mathrm{Fe}$ doped $\mathrm{ZnO}$ fibers were investigated using superconducting quantum interference device (SQUID) magnetometry at temperatures ranging from $2 \mathrm{~K}$ up to 
room temperature. Magnetic Force Microscopy (MFM) (Veeco, Nanoscope IIIa MultiMode with standard MESPtype tip) was applied to image the magnetic signal at room temperature. Prior to MFM the atomic force microscopy (AFM) in tapping mode (Nanoscope IIIa with OTESPA $160 \mu \mathrm{m}$ tip) was used to check the vertical corrugations of the deposited fibers. MFM was performed in lift mode with lift height $150 \mathrm{~nm}$, above the diameter of the thickest fibers.

\section{Results and discussion}

SEM images show entwined NFs with diameters in the range from 100 to $500 \mathrm{~nm}$ and suggest that NFs consist of nanometer size crystallites (Fig. 1). The XRD patterns (Fig. 2) of both undoped and $\mathrm{Fe}$ doped $\mathrm{ZnO}$ nanofibers exhibit peaks revealing their wurtzite structure [33]. No additional peaks are found in the spectra of Fe doped NFs suggesting an absence of sizable precipitates of Fe-rich phases. This observation is supported by TEM investigations. The electron diffraction patterns, shown in Fig. 3, correspond to the wurtzite structure of $\mathrm{ZnO}$ [33]. No evident differences in the diffraction rings were noted pointing out that the doping does not affect the crystalline structure. Importantly, TEM images confirm that the NFs are indeed built as conglomerates of nanocrystallites. The crystal diameters were obtained from dark field TEM images (example inset in Fig. 4a, b). The size distributions for the undoped and Fe doped samples are shown in Fig. 4. For both samples we find average grain size of about $4.5 \mathrm{~nm}$. We did not observed the second phase crystal with

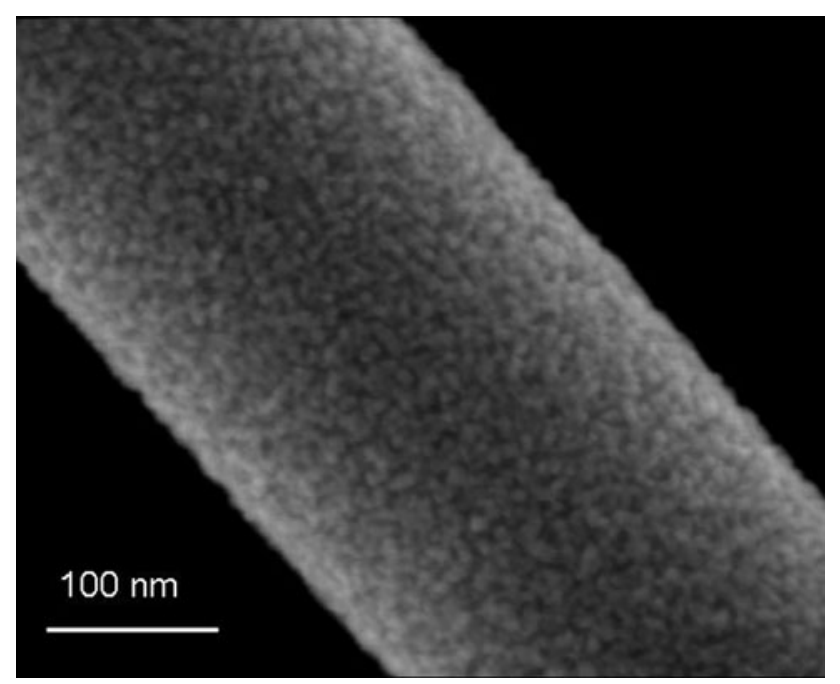

Fig. 1 SEM image of the Fe doped $\mathrm{ZnO}$ nanofiber showing that is formed by the conglomerates of nanocrystallites few nanometer in diameter

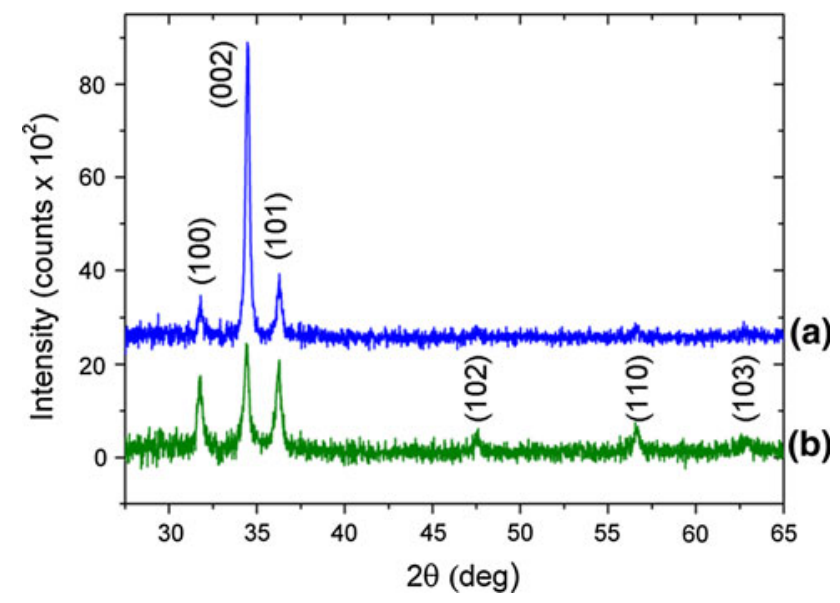

Fig. 2 XRD patterns of $\mathrm{ZnO}$ nanofibers: undoped $(a)$ and Fe doped (b). The top spectrum is shifted by 2,500 counts for clarity

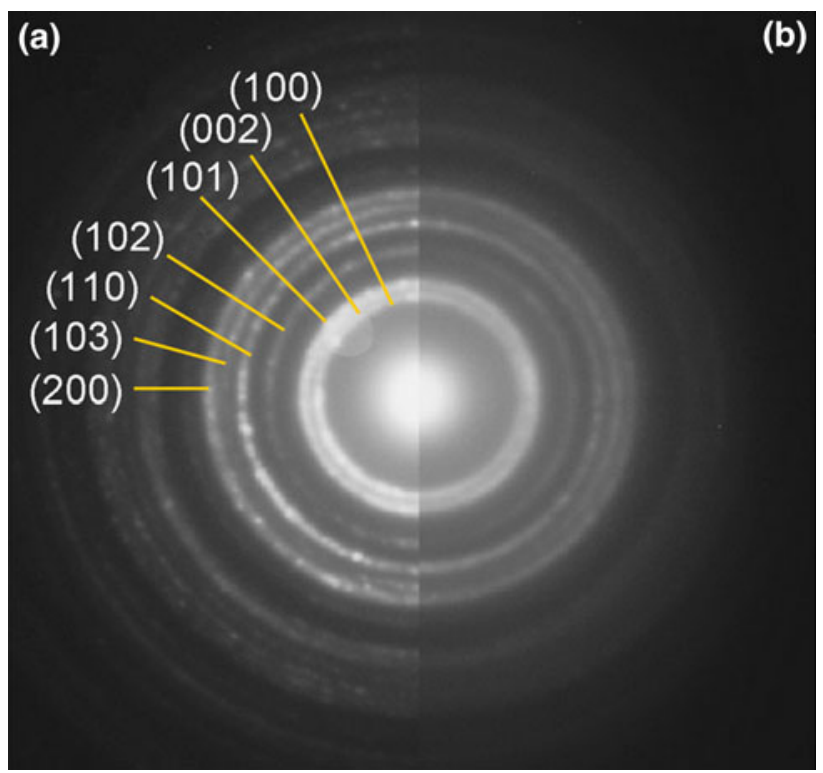

Fig. 3 Electron diffraction pattern of $\mathrm{ZnO}$ nanofibers, undoped (a) and Fe doped (b)

different sizes for Fe doped NFs. The orientation of the nanocrystallites can be inferred from the intensities of XRD and electron diffraction patterns. Among the XRD peaks, the strongest ones are those corresponding to 002 planes (Fig. 2). Moreover, the intensities of electron diffraction rings are not uniform azimuthally (Fig. 3). Both results point out that nanocrystallites are preferentially oriented with the 001 direction perpendicular to the fiber axis.

Chemical characterization was performed by EDX analysis, which confirmed the intended chemical composition of the fibers after electrospinning and calcinations processes. Indeed, a standardless quantitative analysis 


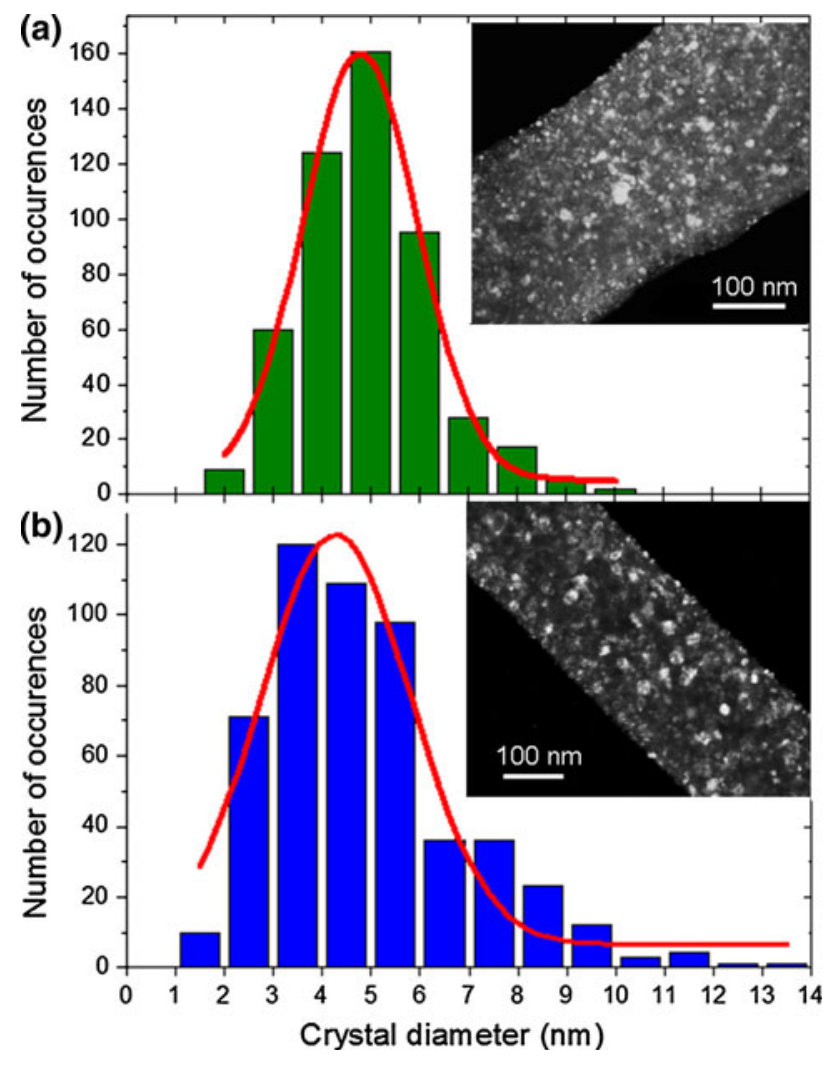

Fig. 4 Crystal size distribution of: Fe doped (a) and undoped $\mathrm{ZnO}$ nanofibers (b) obtained from the TEM measurements. The dark field images: Fe doped (a) and undoped (b) $\mathrm{ZnO}$ nanofibers, respectively (inset)

yielded $\mathrm{Fe} / \mathrm{Zn}$ ratio equal to 0.095 ( \pm 0.012 ). Moreover, the cross-section analysis of a doped sample shows that iron dopant atoms are homogeneously distributed across the nanofibers (Fig. 5). Thus, the EDX analysis supports the conclusion about an absence of sizable $\mathrm{Fe}$ aggregations inside the bulk of the $\mathrm{Fe}$ doped $\mathrm{ZnO}$ nanofibers.

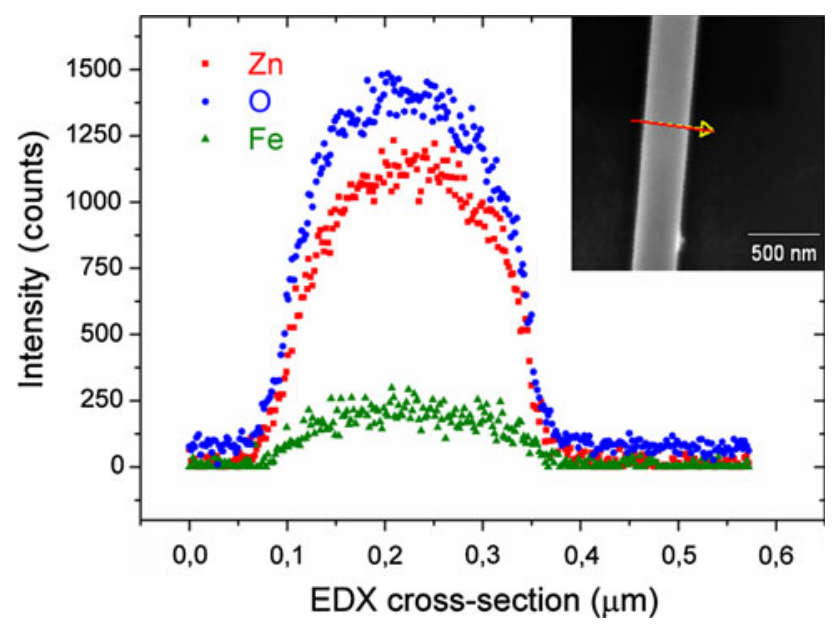

Fig. 5 The EDX signal for $\mathrm{Zn}, \mathrm{O}$ and $\mathrm{Fe}$ atoms across $\mathrm{Fe}$ doped $\mathrm{ZnO}$ nanofiber. Homogenous distribution of $\mathrm{Fe}$ atoms is proved
In Fig. 6, we show a SEM image and a corresponding panchromatic CL map obtained on Fe doped NFs. We find that the light is emitted uniformly from all the fibers. CL (see inset to Fig. 6c) and photoluminescence spectra (not shown) consists of two emission bands. One, centered around $400 \mathrm{~nm}$, is related to the interband recombination. The other one centered around $600 \mathrm{~nm}$ is a defect band. It is present in both doped and undoped fibers and most probably originates from oxygen vacancies [34].

In Fig. 7, we present magnetization dependence on magnetic field measured at various temperatures for $\mathrm{Fe}$ doped nanofibers oriented along the applied field in the magnetometer. Hysteresis loops are clearly seen up to room temperatures (see inset in Fig. 7) indicating a ferromagnetic coupling present in the system. Both, the magnetic signal and coercive field decreasing on temperature. At room temperature, we still observe a rapid magnetic response to the applied magnetic field. The magnetic signal saturates at above about 5000 Oe. Such a shape of the hysteresis loop clearly indicates that the Curie temperature $\mathrm{T}_{\mathrm{C}}$ is substantially larger than $300 \mathrm{~K}$.

At temperatures below $50 \mathrm{~K}$, we find that the magnetic signal does not saturate in magnetic fields below $10 \mathrm{kOe}$. It is a clear indication that a paramagnetic phase is also present in the NFs. This paramagnetic signal most likely originates from substitution $\mathrm{Fe}^{2+}$ and therefore demonstrates a formation of a $(\mathrm{Zn}, \mathrm{Fe}) \mathrm{O}$ ternary alloy.

Figure 8 shows the $30 \mu \mathrm{m} \times 30 \mu \mathrm{m}$ topography and corresponding MFM image (taken at $150 \mathrm{~nm}$ lift height) of several randomly oriented $\mathrm{Fe}$ doped $\mathrm{ZnO}$ fibers, revealing that majority of the fibers interacts magnetically with the MFM probe. Attractive magnetic interactions (dark regions) are clearly visible as shifts in tip resonance frequency as high as few Hz. We find that the magnetic signal is related solely to the fibers and that the Fe ions are evenly distributed along the fibers, but since their magnetization is softer than that of the tip material, only attractive MFM contrast is observed.

The data in Fig. 7, as well as results of zero field cooled (ZFC) and field cooled (FC) measurements (presented in Fig. 9), suggest that the ferromagnetic signal is due to a cooperative behavior typical to a blocked superparamagnet. This indicates that the ferromagnetic coupling takes place only locally. This is not surprising since the structural studies reveal that the NFs consist of nanometer scale crystallites. Their blocked state at room temperature is further corroborated by the presence of a non-zero coercivity of about 60 Oe (see inset in Fig. 7). In order to estimate the volume of magnetic nanoparticles giving rise to the room temperature ferromagnetism, we employ the common formula connecting the value of the blocking temperature $T_{B}$ with the particle volume $V_{B}$ and magnetic anisotropy constant $\mathrm{K}$ for conventional magnetometry: 
Fig. 6 SEM (a), CL panchromatic (b) and CL at $400 \mathrm{~nm}(\mathbf{c})$ images of Fe doped $\mathrm{ZnO}$ nanofibers. Inset: $\mathrm{CL}$ spectrum Fe doped nanofibers
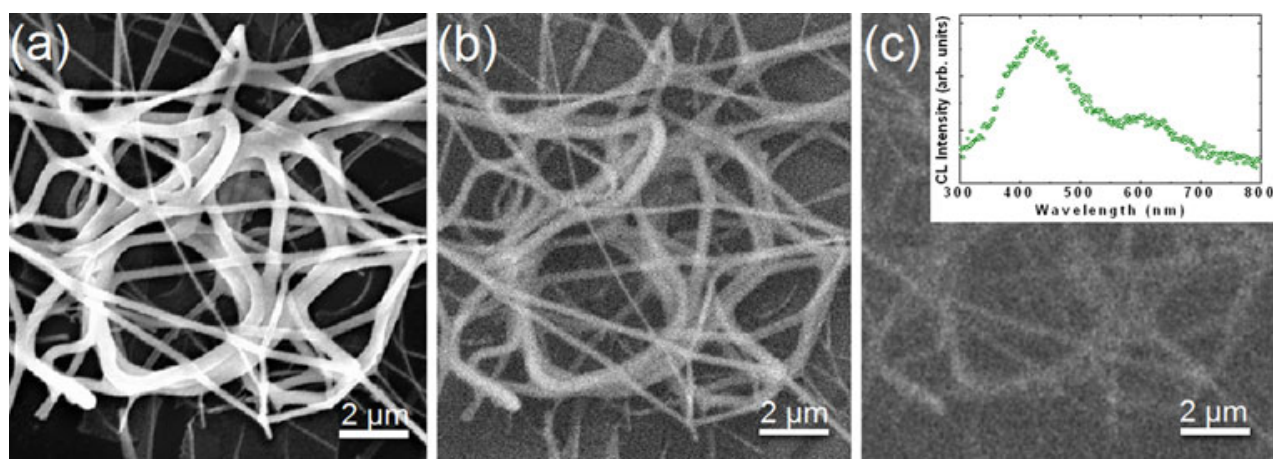

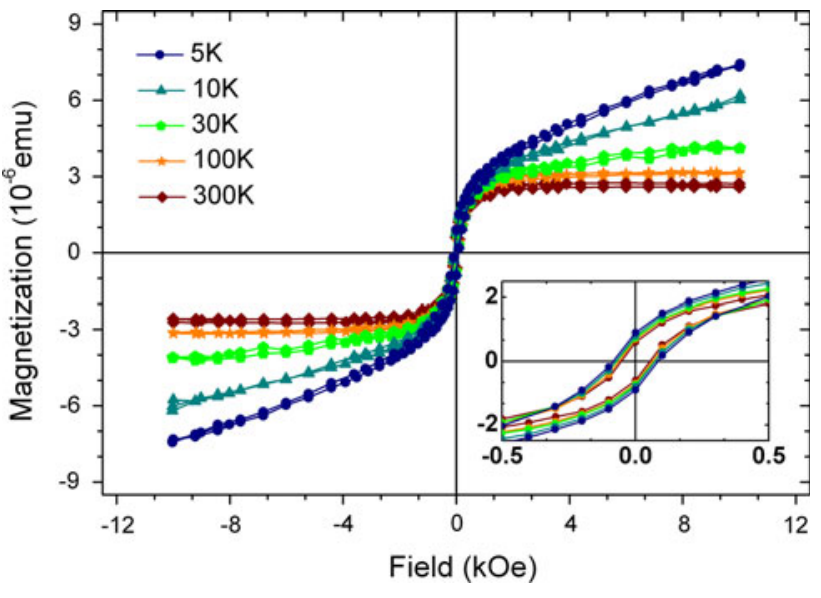

Fig. 7 Effect of magnetization on magnetic field of Fe doped $\mathrm{ZnO}$ nanofibers oriented along the applied magnetic field in the magnetometer for various temperatures. Enlargement of the hysteresis loops in the -0.5 to $0.5 \mathrm{kOe}$ magnetic field range (inset)

$T_{B}=25 k_{B} V_{B} / K$, where $k_{B}$ is the Boltzman constant. For our material, the anisotropy constant is unknown so we take $\mathrm{K}$ in range between $10^{5}$ and $10^{6} \mathrm{erg} / \mathrm{cm}^{3}$, which spans the range of values for bulk crystals (like $\mathrm{Fe}$ or its oxides) and nanoparticles with surface enhanced anisotropy. For $\mathrm{T}_{\mathrm{B}}=300 \mathrm{~K}$, we obtain nanoparticle diameters between 3 and $10 \mathrm{~nm}$. Notably, such particle diameters very well agree with nanocrystal sizes obtained from TEM studies.
Interestingly, a weak signal at remanence (approximately $1 / 4$ of that in saturation) indicates that the majority of the magnetic nanoparticles in this ensemble is probed along their hard axis. Given that during the measurement the vast majority of the nanofibers was oriented along the magnetic field, we conclude that these nanoparticles have their magnetic easy axes preferentially oriented radially to the axis of the fibers. Moreover, the data collected in Fig. 9 shows a clear peak on ZFC measurement that defines another source of magnetic response in this system with a low blocking temperature of $T_{B} \cong 20 \mathrm{~K}$. It points out that there is another kind of magnetic nanoparticles present in the fibers. Using the same arguments as above, we find the corresponding diameters of these nanoparticles to be in the range between 3 and $1 \mathrm{~nm}$ for a bulk or the surfaceanisotropy enhanced $\mathrm{K}$, respectively.

We do not have all, direct or definitive evidence which mechanism is responsible for the observed ferromagnetism in our NFs. However we are inclined to the following two possibilities. The first one is related to $\mathrm{Fe}$ ions substitution in the $\mathrm{ZnO}$ matrix. These ions can form a robust ferromagnetic phase mediated by delocalized p-type carriers (holes) [1] or through a superexchange with oxygen vacancies [18]. Since typically $\mathrm{ZnO}$ exhibits an n-type conductivity, we conclude that the latter mechanism is the more probable one. Indeed, recently the crucial role of

Fig. 8 Comparison of the AFM (a) and the MFM (b) images of Fe doped $\mathrm{ZnO}$ nanofibers. The scanned areas for both images are $30 \mu \mathrm{m} \times 30 \mu \mathrm{m}$
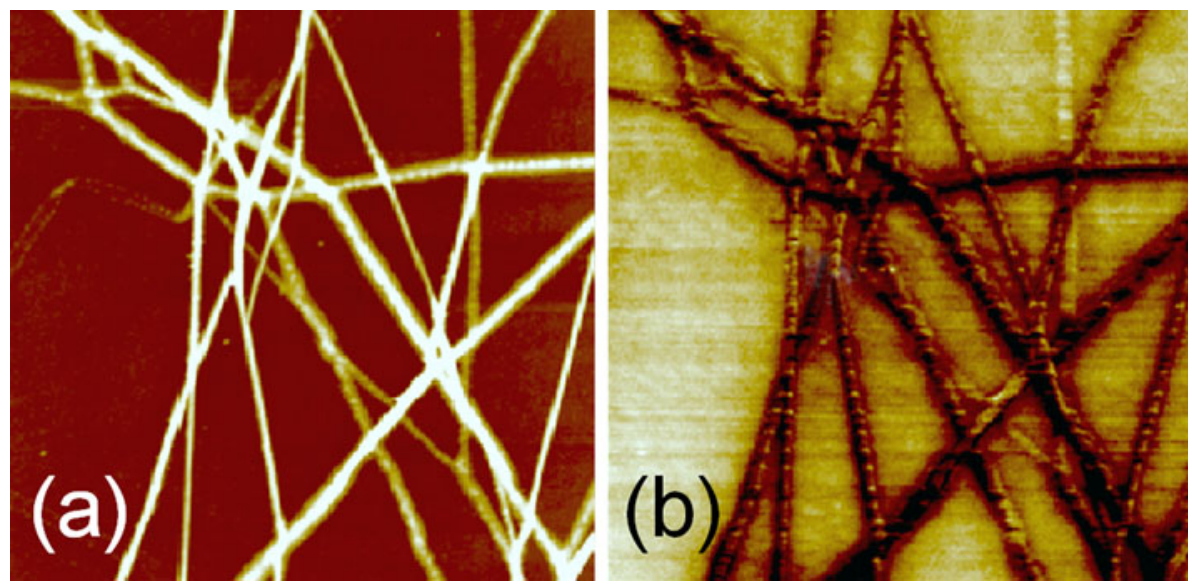


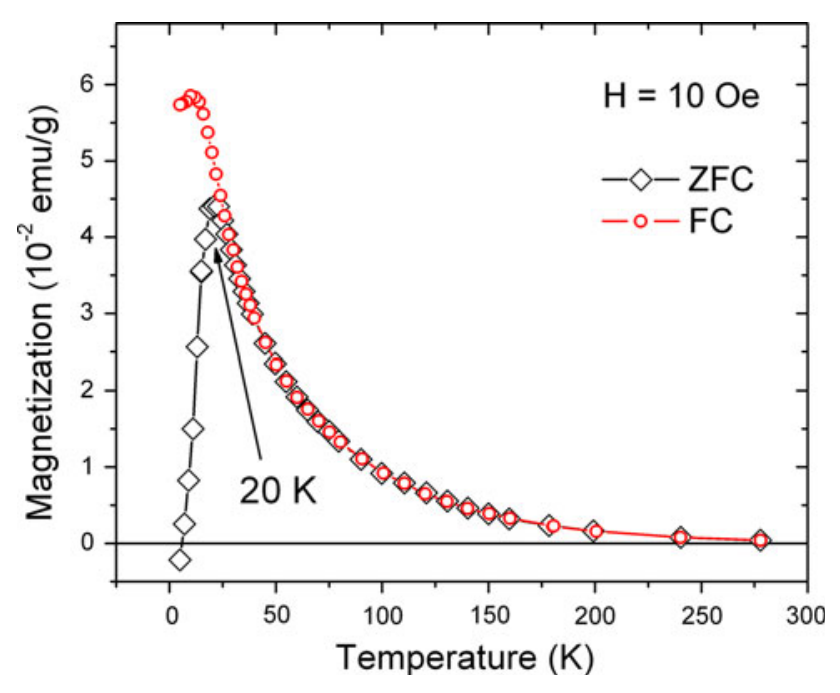

Fig. 9 Zero-field-cooled and field-cooled magnetization as a function of temperature for Fe doped $\mathrm{ZnO}$ nanofibers

oxygen vacancies in appearance of the ferromagnetic phase has been proved experimentally [18]. We note that during the calcinations process the presence of carbon ions from precursor substances facilitate the formation of oxygen vacancies in our samples. The second possible explanation is related to a nanoscale phase separation responsible for the presence of Fe-rich magnetic nanoparticles [35]. These particles can take form of $\alpha$-Fe, various Fe-oxides and/or condensed ( $\mathrm{Zn}, \mathrm{Fe}) \mathrm{O}$. These particles can generate a strong ferromagnetic signal and remain not detected by our structural characterization methods.

\section{Conclusions}

In conclusion, we developed a fabrication method, which allows us to obtain nanofibers exhibiting ferromagnetism at room temperature. By electrospinning and calcination, we produce about $200 \mathrm{~nm}$ in diameter Fe doped $\mathrm{ZnO}$ fibers of virtually infinite length, build of about $4.5 \mathrm{~nm}$ crystallites oriented with 001 crystal axis perpendicular to the fiber axis. We demonstrate that some of the $\mathrm{Fe}$ atoms substitute $\mathrm{Zn}$ in the $\mathrm{ZnO}$ matrix and give rise to a paramagnetic signal at low temperatures. Incorporating $10 \% \mathrm{Fe}$ ions does not modify the wurtzite crystal structure of the host $\mathrm{ZnO}$. While the exact origin of the room temperature ferromagnetism remains unknown, we suggest that it is either due to a mechanism mediated by presence of oxygen vacancies or related to small precipitates of ferromagnetic phases of iron. Importantly, compared to previously reported $\mathrm{Co}$ and $\mathrm{Mn}$ doped $\mathrm{ZnO}$ based semiconductors, the synthesized material is a good candidate for biologically compatible therapeutic and diagnostic applications.
Acknowledgments The research was partially supported by the European Union within European Regional Development Fund, through Grant Innovative Economy (POIG.01.01.02-00-008/08) and by the Ministry of Science and Higher Education (Poland) through Grant No. N518 424036 and No. N515 015 32/0997.

Open Access This article is distributed under the terms of the Creative Commons Attribution Noncommercial License which permits any noncommercial use, distribution, and reproduction in any medium, provided the original author(s) and source are credited.

\section{References}

1. Dietl T, Ohno H, Matsukura F, Cibert J, Ferrand D (2000) Science 287:1019-1022

2. Venkatesan M, Fitzgerald CB, Coey JMD (2004) Nature 430:630

3. Coey JMD, Venkatesan M, Fitzgerald CB (2005) Nat Mater 4:173-179

4. Seshadri R (2005) Curr Opin Solid State Mater Sci 9:1-7

5. Yang JH, Zhao LY, Zhang YJ, Wang YX, Liu HL (2008) Cryst Res Technol 43(9):999-1003

6. Risbud AS, Spaldin NA, Chen ZQ, Stemmer S, Seshadri R (2003) Phys Rev B 68:205202

7. Prellier W, Fouchet A, Mercey B, Simon Ch, Raveau B (2003) Appl Phys Lett 82:3490-3492

8. Lim S-W, Hwang D-K, Myoung J-M (2003) Solid State Commun 125:231-235

9. Lee H-J, Jeong S-Y (2002) Appl Phys Lett 81:4020-4022

10. Sati P, Hayn R, Kuzian R, Régnier S, Schäfer S, Stepanov A, Morhain C, Deparis C, Laügt M, Goiran M, Golacki Z (2006) Phys Rev Lett 96:017203

11. Liu H, Yang J, Zhang Y, Yang L, Wei M, Ding X (2009) J Phys: Condens Matter 21:145803

12. Hong NH, Sakai J, Brizé V (2007) J Phys: Condens Matter 19:036219

13. ACh Mofor, El-Shaer A, Bakin A, Waag A (2005) Appl Phys Lett 87:062501

14. Sundaresan A, Bhargavi R, Rangarajan N, Siddesh U, Rao CNR (2006) Phys Rev B 74:161306R

15. Kim JH, Kim H, Kim D, Yoon SG, Choo WK (2004) Solid State Commun 131:677-680

16. Yoon SW, Cho S-B, We SC, Yoon S, Suh BJ (2003) J Appl Phys 93:7879

17. Lee E-C, Chang K (2004) J Phys Rev B 69:085205

18. Samariya A, Singhal RK, Kumar S, Xing YT, Alzamora M, Dolia SN, Deshpande UP, Shripathi T, Saitovitch EB (2010) Mater Chem Phys 123:678-684

19. Cho YC, Kim S-J, Lee S, Kim SJ, Cho CR, Nahm H-H, Park CH, Jeong K, Park S, Hong TE, Kuroda S, Jeong S-Y (2009) Appl Phys Lett 95:172514

20. Huang Z-M, Zhang Y-Z, Kotaki M, Ramakrishna S (2003) Comp Sci Technol 63:2223-2253

21. Li D, Xia Y (2004) Adv Mater 16(14):1151-1170

22. Ramaseshan R, Sundarrajan S, Jose R, Ramakrishna S (2007) J Appl Phys 102:111101

23. Wu H, Lin D, Zhang R, Pan W (2008) J Am Ceram Soc 91(2):656-659

24. Baranowska-Korczyc A, Sikora B, Zaleszczyk W, Nowicka A, Fronc K, Wojciechowski T, Dziawa P, Knoff W, Gas K, Paszkowicz W, Szuszkiewicz W, Świątek K, Kłopotowski Ł, Sobczak K, Dłużewski P, Karczewski G, Wojtowicz T, Bujak J, Elbaum D (2009) ICFSI-12 in Weimar, abstract-book 302

25. Park J, Kim SS (2009) J Am Ceram Soc 92(8):1691-1694 
26. Yang X, Shao C, Guan H, Li X, Gong J (2004) Inorg Chem Commun 7:176-178

27. Ren H, Ding Y, Jiang Y, Xu F, Long Z, Zhang P (2009) J Sol-Gel Sci Technol 52:287-290

28. Pan F, Song C, Liu XJ, Yang YC, Zeng F (2008) Mater Sci Eng R $62: 1-35$

29. Li Z, Yang R, Yu M, Bai F, Li Ch, Wang ZL (2008) J Phys Chem C 112:20114-20117
30. Gopikrishnan R, Zhang K, Ravichandran P, Baluchamy S, Ramesh V, Biradar S, Ramesh P, Pradhan J, Hall JC, Pradhan AK, Ramesh GT (2010) Nano-Micro Lett 2(1):31-36

31. Gupta AK (2005) Biomaterials 26(18):3995-4021

32. Li D, Wang Y, Xia Y (2003) Nano Lett 3(8):1167-1171

33. Data Base: JCPDS, file 36-1451

34. Cheng W, Ma X (2009) J Phys: Conf Ser 152:012039

35. Bonanni A, Dietl T (2010) Chem Soc Rev 39:528-539 